\title{
Quantitative measurement of reactive oxygen species in vivo by utilizing a novel method: chemiluminescence with an internal fluorescence as reference
}

\author{
Yanchun Wei \\ Da Xing \\ Shiming Luo \\ Liyong Yang \\ Qun Chen \\ South China Normal University \\ College of Biophotonics \\ MOE Key Laboratory of Laser Life Science \\ and \\ Institute of Laser Life Science \\ Guangzhou, 510631 China
}

\begin{abstract}
Reactive oxygen species (ROS) produced by photodynamic therapy (PDT) is recorded in vivo using a chemiluminescence (CL)based gated optical system. A novel approach is developed to utilize the fluorescence $(\mathrm{FL})$ of the $\mathrm{CL}$ probe as an internal fluorescence to calibrate the observed CL on pharmacokinetics of the probe in situ. The results show that during an in vivo PDT session, the intensity of $\mathrm{CL}$ decreases significantly and the decaying of $\mathrm{CL}$ is governed by fast and slow time components. By comparing the temporal profile of $\mathrm{FL}$ to that of the corresponding $\mathrm{CL}$, it is found that the slow component is mainly attributed to the probe pharmacokinetics, whereas the fast component is likely due to rapid oxygen consumption as a result of PDT treatment. With carefully selected criteria, it is possible to minimize the effect of probe pharmacokinetics. This significantly improves the monitoring method for practical applications. $\odot 2010$ Society of PhotoOptical Instrumentation Engineers. [DOI: 10.1117/1.3368688]
\end{abstract}

Keywords: reactive oxygen species; chemiluminescence; fluorescence; photodynamic therapy; in vivo.

Paper 09493R received Nov. 7, 2009; revised manuscript received Feb. 3, 2010; accepted for publication Feb. 3, 2010; published online Mar. 25, 2010.

\section{Introduction}

Reactive oxygen species, especially singlet oxygen $\left({ }^{1} \mathrm{O}_{2}\right)$, are important cytotoxins. ${ }^{1,2}$ They can depress cell activity and even induce cell death by oxidizing lipids, proteins, and DNA. Photodynamic therapy (PDT) utilizes light to activate a preadministered photosensitizer drug to photochemically produce reactive oxygen species for a localized cytotoxicity. ${ }^{3,4}$ Aside from the intrinsic tissue response to the therapy, there are three main factors governing the effectiveness of a PDT treatment: photosensitizer concentration in the target, light absorption by the photosensitizer, and availability of molecular oxygen at the treatment site. ${ }^{2}$ Although each of these factors can be controlled to a certain extent in order to manipulate the treatment outcome, the interplay among them is complex and individual dosimetry for each component in most cases is empirical and does not do justice to the precision required for most clinical cases. This is certainly the case if a PDT treatment involves sensitive organs in which PDT may have advantages due to its high localization property. Because reactive oxygen species are a well-defined cytotoxic agent mediating PDT damage, it is reasonable to monitor them as a means for the PDT dosimetry. ${ }^{1,5,6}$

The chemiluminescence (CL) technique is usually used to evaluate free radicals and other biologic or pharmacologic

Address all correspondence to Da Xing, MOE Key Laboratory of Laser Life Science \& Institute of Laser Life Science, College of Biophotonics, South China Normal University, Guangzhou 510631, China. Fax: 86-20-85216052; Tel: 8620-85210089; E-mail: xingda@scnu.edu.cn molecules. ${ }^{7-9}$ Several CL probes have been synthesized to monitor reactive oxygen species (ROS) production. Most ROS-specific CL probes are related to fluorescein or its ana$\log$ and are commercially available as a synthesized product. ROS can chemically interact with probe molecules, which results in certain changes in their conformation. In the process, the probe molecules reach a higher/excited energy state(s), and then, during the subsequent de-excitation, photons in the visible wavelength range are emitted as CL and can be detected with conventional optical apparatus. ${ }^{10}$

There are several CL probes that have been used to evaluate ROS production due to their high selectivity, sensitivity, easy detection, and feasibility of usage for in vivo studies. ${ }^{11-13}$ In the current study, we employed fluorescenyl cypridina luciferin analog [(FCLA), [3, 7-dihydro-6- [4-[2-[N'-(5-fluoresceinyl) thioureido] ethoxy] phenyl]-2-methylimidazo $[1,2$-a] pyrazin-3-one], which can selectively detect singlet oxygen and superoxide $\left(\mathrm{O}_{2-}\right)$ and produce photons at $532 \mathrm{~nm}^{14}$ We have previously reported that the probe can be easily absorbed by cells with minimal toxicity and the CL emission can be detected with a conventional photon multiplier tube. ${ }^{15}$ The probe has its optimum detection efficiency at $\mathrm{pH}$ 7.4, which is in accordance with physiological conditions. ${ }^{16}$

Currently, there are several PDT dosimetry methods, including the electron spin resonance method and fluorescence (FL) probe method are available; they are in general limited

$1083-3668 / 2010 / 15(2) / 027006 / 7 / \$ 25.00$ ○ 2010 SPIE 
either by complex technology or high cost for practical applications. ${ }^{6}$ CL method has its own advantages: high selectivity, sensitivity, easy operation, and potential practical application for in vivo studies. As an alternative method for PDT dosimetry, the feasibility of using FCLA as a PDT dosimeter was previously reported by this laboratory. ${ }^{17}$ In the preliminary study, it was reported that the cumulated CL during a PDT treatment is directly related to the biological outcome, regardless of the treatment protocol (irradiation light fluence, photosensitizer concentration, etc.). Yet, FCLA is subjected to its own pharmacokinetics in the target tissue. If it is to be used as a practical sensor for PDT dosimetry, it is important to understand how this pharmacokinetics affects the reliability of the measurement.

CL signal alone can be influenced by many factors in the dynamic process, such as target oxygen consumption, photosensitizer bleaching, and acute vessel damage as a result of the treatment, which can all contribute to change the CL signal intensity, and this portion of the change is directly linked to the production of ROS and serves as our intended dosimetry marker. On the other hand, the dynamic process of probe consumption as a result of photochemical reaction and, more importantly, pharmacokinetics of the probe (tissue uptake and elimination), also contribute to the observed CL intensity and cause misinterpretation of ROS production. CL should thus be carefully evaluated and corrected for a practical and more precise ROS measurement. Clearly, CL measurement alone cannot provide adequate information to solve the puzzle. In the current study, a novel optical biosensor system is developed for simultaneous monitoring of both CL and its corresponding probe FL. The principle of this sensor system is analyzed and tested for in vivo ROS measurement. The results show that in situ ROS monitoring can be reliably achieved with minimal interference to the PDT process. The pharmacokinetics of the ROS probe can be compensated for with simultaneous FL measurement, thus, significantly simplifying the monitoring process without sacrificing its reliability.

\section{Materials and methods}

\subsection{Theory for Measurement of $C L$ and FL}

\subsubsection{Detection reactive oxygen with $C L$}

CL signal intensity is much weaker than that of excitation light. Although it is technically feasible to use an optical system to resolve a CL signal from its excitation light, given that most CL probes are known to have much longer lifetimes compared to that of fluorescence, it is much easier to measure CL by turning off the excitation light temporarily to completely eliminate the interference of the excitation light. This provides maximized signal-to-noise ratio of the measurement and, at the same time, protects the optical detection system [typically, photon multiplier tubes (PMTs)] from overloading. To verify that the delayed measurement is directly linked to ROS production, the deexcitation process of CL can be expressed as

$$
i_{\mathrm{d}}(t)=I_{\mathrm{CL}} e^{-t / \tau},
$$

where $I_{\mathrm{CL}}$ and $i_{\mathrm{d}(t)}$ are CL fluence rate at time that illumination is ceased and at decay time $t$, respectively; $\tau$ is lifetime of CL.
Assuming that the lifetime of the CL probes remain constant for each treatment protocol, CL acquired at a consistent time $t$ can be expressed as

$$
I_{\mathrm{d}}=\beta I_{\mathrm{CL}}
$$

where $\beta$ is a constant determined by the lifetime $\tau$ and the time $t$ (in the current study, this is the delay time between irradiation light off and the starting of CL). The equation indicates that, as long as a consistent delay time is used, the acquired CL can be used to express the fluence of CL. Because CL is directly proportional to ROS production, measurement made at a delayed time $t$ therefore is also directly linked to the ROS production,

$$
I_{\mathrm{d}} \propto[\mathrm{ROS}] .
$$

This establishes that a delayed CL measurement provides reliable information of ROS production.

\subsubsection{Detection in vivo elimination rate with $F L$}

Relationship of FL and probe concentration can be expressed by FL theory and Beer-Lambert theory as

$$
\mathrm{FL}=K c \quad\left\lfloor K=2.3 I_{0} \varepsilon_{(\lambda A)} l \phi Z\right\rfloor,
$$

where $c$ is probe concentration, $I_{0}$ is the intensity of irradiation light, $\varepsilon_{(\lambda A)}$ is extinction coefficient, $l$ is radiation distance, $\phi$ is quantum efficiency, and $Z$ is instrument factor.

Thus, the FL of probe in vivo can be described as

$$
\begin{gathered}
k \mathrm{FL}_{t} d t-\left(K_{2}-K_{1}\right) f(t) d t=d \mathrm{FL}_{t} \\
k=\frac{\left(K_{2}-K_{1}\right) f(t)-\left(\mathrm{FL}_{t}\right)^{\prime}}{\mathrm{FL}_{t}},
\end{gathered}
$$

where the $\mathrm{FL}_{t}$ is fluorescence intensity detected at $t ; k$ is elimination rate constant; $K_{1}$ is CL probe FL intensity coefficient before reaction and $K_{2}$ is CL probe oxide FL intensity coefficient; $f(t)$ is the oxidated CL probe concentration of $t$ moment and

$$
f(t)=\rho \int_{t_{1}}^{t_{2}} I_{\mathrm{d}}(t) d t,
$$

where $I_{d}(t)$ is $t$ moment CL intensity recorded by system, and $\rho$ is the relation coefficient of $I_{d}(t)$ and $f(t)$.

\subsubsection{Correction $C L$ in vivo}

On the basis of photochemical theory, the concentration of $\mathrm{CL}$ probe changes by depletion as

$$
-d c_{t}=k c_{t} d t+f(t) d t,
$$

where $c_{t}$ is CL probe concentration at $\mathrm{t}$ moment.

The result is

$$
c_{t}=\left[c_{0}-\int_{0}^{t} f(t) e^{k t} d t\right] e^{-k t},
$$

where $c_{0}$ is primary concentration of CL probe. 
Because the CL detection is not continuous, in order to calculate $c_{t}$, the Eq. (9) at a detection interval can be closely written as

$$
c_{t} \approx c_{t 0} e^{-k \Delta t}-f(t) \Delta t,
$$

where $\Delta t$ is time interval, decided by a selected experiment parameter and $c_{t 0}$ is the initial concentration of every interval. Thus in our system, the FCLA-CL can be corrected by

$$
I_{\mathrm{CL}^{\prime}}=\frac{c_{0}}{c_{t}} I_{\mathrm{CL}}
$$

\subsection{Chemicals and Materials}

The ROS-specific CL probe, FCLA (Free Acid FCLA, Tokyo Kasei Kogyo Co., Tokyo, Japan) was dissolved in doubledistilled water $(100 \mu \mathrm{M})$ and stored at $-80{ }^{\circ} \mathrm{C}$. The probe produces a $532-\mathrm{nm} \mathrm{CL}$ and is at its maximum detection efficiency in biological $\mathrm{pH}$ range. For the photosensitization reaction, photosensitizer Protoporphyrin IX disodium salt (Aldrich Chemical Co., Milwaukee, Wisconsin) was prepared as 200- $\mu \mathrm{M}$ stock solution and stored in the dark at $4{ }^{\circ} \mathrm{C}$ until needed.

\subsection{Detection}

In preliminary study, it was found that FCLA could emit CL due to self-oxidation. The signal intensity increased with the increase of FCLA concentration. This relationship, in conjunction with a simulated tissue phantom made with India ink and Lipovenoes (PH. 7.4, $\mu_{\mathrm{a}}=1.67 \mathrm{~mm}^{-1}, \mu_{\mathrm{s}}=2.6 \mathrm{~mm}^{-1}$, $532 \mathrm{~nm}$.), was used to estimate the initial tissue concentration of FCLA. A range of FCLA concentration (2.5, 5, 10, $20 \mu \mathrm{mol} / \mathrm{L}$ ) was mixed into the optical phantom, and the corresponding spontaneous CL intensity was measured for the titration purpose. Each experiment was repeated three times.

\subsection{In Vivo CL and FL Measurements}

BALB/ca mice (both gender, average weight $\sim 20 \mathrm{~g}$, Center of Experimental Animal SunYat-sen University, Guangzhou, China) were used to provide an in vivo normal skin model. The animals were housed in a standard animal-keeping facility with controlled temperature and 12/12-h light cycle. Before each experiment, the hind leg of a BALB/ca mouse was molted with depilatory $\left(\mathrm{Na}_{2} \mathrm{~S} 8 \%\right.$ aqua solution). The mice were divided into three groups according to the irradiation fluence rate $\left(10,30\right.$, and $\left.50 \mathrm{~mW} / \mathrm{cm}^{2}\right)$, and there were seven mice in every group. Premixed PPIX $(0.02 \mu \mathrm{mol})$ and FCLA $(0.01 \mu \mathrm{mol})$ in $200 \mu \mathrm{L}$ physiologic saline were injected subcutaneously into the molted leg, $1 \mathrm{~h}$ prior to the PDT light irradiation. This time delay allowed the drug to reach a stable concentration distribution in the target. ${ }^{18,19}$ The time sequence of the signal acquisition was controlled with the computer program described above: the irradiation light was interrupted every $2 \mathrm{~s}$ for 1 -s duration to collect CL.

In order to investigate the relationship between $\mathrm{CL}$ and oxygen depletion during PDT, a long-interval irradiation was carried out. Drugs were employed in the same manner as above and injected into mouse skin. The fluence rate was $50 \mathrm{~mW} / \mathrm{cm}^{2}$. After a 60 -s irradiation time with simultaneous
CL recording, the tissue was allowed 180-s dark time preceding the next illumination. This procedure was repeated nine times.

For FL detection, FCLA FL was excited by a 488-nm laser $\left(5 \mu \mathrm{W} / \mathrm{cm}^{2}\right)$. The filters $(510$ and $530 \mathrm{~nm}$ bandpass (BP) filter, Oriel Co., Stratford, Connecticut) were used to protect PMTs and transfer photons. The filter can be switched between 510 and $530 \mathrm{~nm}$ during detection of CL and FL. The drugs were used in the same manner as described above. For PDT treatment, the target area was irradiated with a diode laser at $20 \mathrm{~mW} / \mathrm{cm}^{2}$. The FL signals were collected for $2 \mathrm{~s}$ after $20 \mathrm{~s}$ of irradiation and repeated. At the same time, gated collection of the CL signals was made as described above.

\subsection{Biosensor System}

A custom-built apparatus was used for integrated PDT treatment and in situ FL/CL measurements (Fig. 1). For PDT treatment, the irradiation light source was a gated diode laser system [635-nm laser diode controller, LDC 2000, ThorLab (Newton, New Jersey) and TEC 2000, Wavelength Electronics (Bozeman, Montana)]. The laser system was controlled and modulated by the transistor-transistor logic (TTL) level of the counter. The excitation light source for the in situ FL measurement was an argon-krypton laser (488 nm, model 5500 ASL, Aiao Laser Co., Shanghai, China), with its output modulated by a mechanical shutter system (Princeton Instruments, Inc., USA). The shutter was controlled via its driver TTL with a response time of $<10 \mathrm{~ms}$.

Both CL and FL signal were collected with a channel photomultiplier dc-module [(CPDM), model MD952, PerkinElmer, Wiesbaden, Germany] and a counter (PCL-1751, Advantech Co., Ltd. Taiwan), after passing through BP filters (510 and $530 \mathrm{~nm}$, for CL and FL signal, respectively). The filters were mechanically selected and set into the optical path by a relay controlled with proper TTL. The PDT light irradiation, FL, and CL measurement were time sequenced, and the synchronization was realized by controlling each component with a centralized computer program (LABVIEW version 8.2, National Instruments, USA). The time sequence of the signal excitation and collection is shown in the inset of Fig. 1. To protect detector overloading, the cathode of the CPDM was switched off and the shutter was closed during PDT light irradiation.

\section{Results}

Figure 2 is a representative CL temporal profile during a PDT irradiation of normal skin. The data are fit with a biexponential decay model. CL decay times of the fast and slow components, defined as the inverse of the exponential decaying constant from data fitting, are 143 and 1040 s, respectively. The inset of Fig. 2 is a representative of the CL signal during PDT and that of control (no photosensitizer). The results clearly demonstrate that, with the current system, a high signal-to-noise ratio for in vivo ROS monitoring can be achieved.

Figure 3 indicates that, given identical irradiation protocol, decay times of fast and slow components of the CL dynamic process are reasonably consistent. Yet, both are affected by the irradiation fluence rate. The CL decay time of both components is longer at low-irradiation fluence rate compared to 


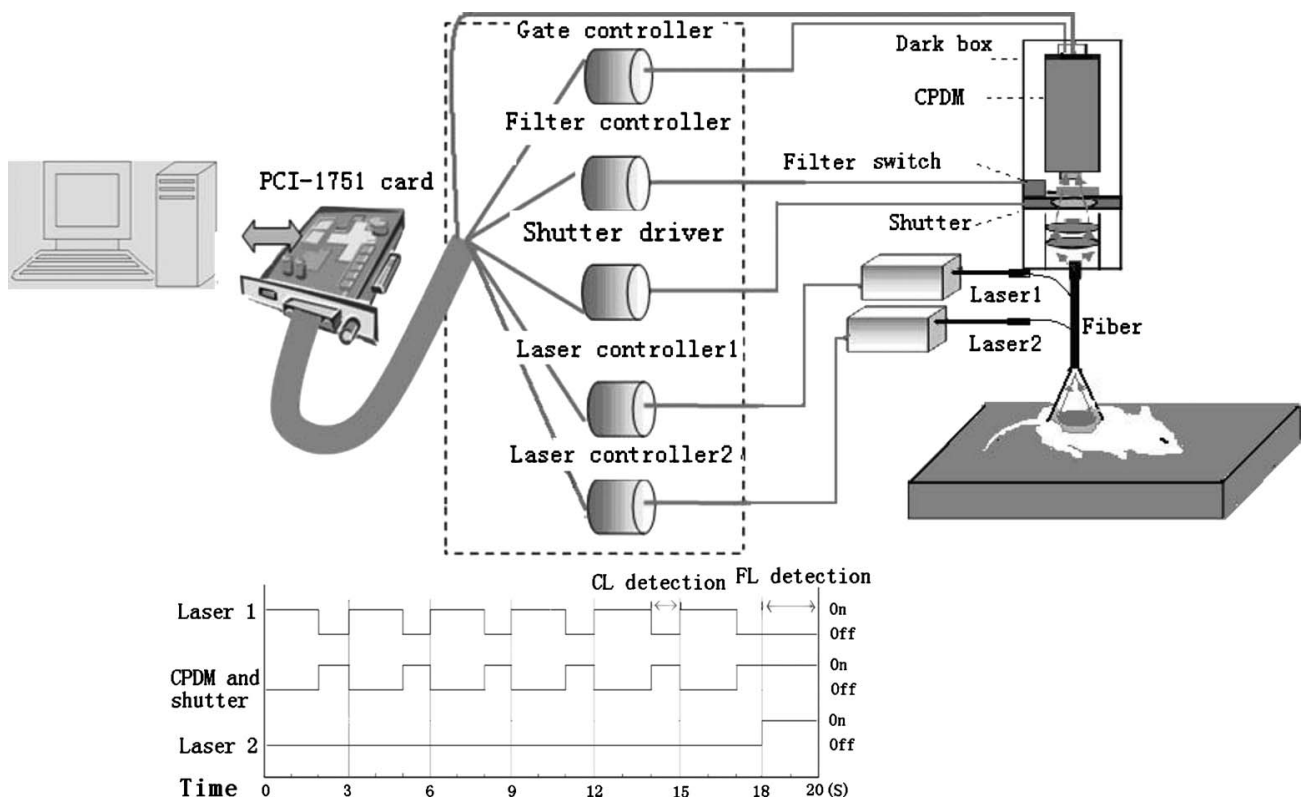

Fig. 1 CL-based reactive oxygen biosensor block diagram illustrating major hardware subsystems. Power supplies omitted for sake of clarity. The timing chart shows CL and FL detection sequence during PDT.

that of the higher ones. However, as the irradiation fluence increases, the decay time becomes more stable (as shown in Fig. 3).

A fractionated irradiation scheme has been used in PDT treatment to compensate for the rapid oxygen depletion during light irradiation. ${ }^{20}$ Although it has been shown that, to a degree, the tactic may compensate for the oxygen depletion and subsequently improve the treatment outcome, its effect on the ROS production has never been explored. In the current study, CL production was measured in situ during a fractionated PDT irradiation (Fig. 4). The result clearly shows that, during each PDT irradiation, CL production decreases rapidly. Yet,

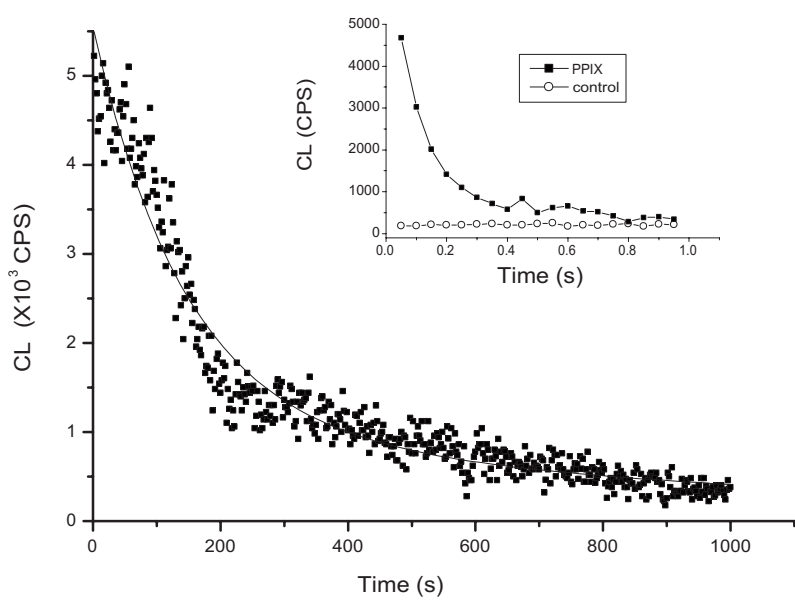

Fig. $2 \mathrm{CL}$ during irradiating mouse that absorbed photosensitizer. Before illumination $1 \mathrm{~h}$ FCLA $(0.01 \mu \mathrm{mol}, 100 \mu \mathrm{L})$ and PPIX $(0.02 \mu \mathrm{mol}, 100 \mu \mathrm{L})$ was injected in situ. Plot shows a representative temporal profile of $\mathrm{CL}$ intensity during PDT $\left(30 \mathrm{~mW} / \mathrm{cm}^{2}, 30 \mathrm{~J} / \mathrm{cm}^{2}\right)$. Fast CL decay time: $143 \mathrm{~s}$; slow CL decay time: $1040 \mathrm{~s}$. In set: $C L$ in one detection circle (50 ms per count). CPS stands for counts per second. with a short 3-min interval, CL production rebounds remarkably to near its previously recorded level. The rapiddecreasing CL during PDT irradiation is dominated by the fast component obtained earlier and is reasonably consistent for each irradiation period throughout the treatment with multiple reperfusions. The extent of CL rebound after each reperfusion varies, likely a result of spontaneous animal movement, but follows a general trend of gradual decrease. Furthermore, the statistical data of initial CL intensity at different PDT doses were also shown in Fig. 5. CL intensity was increased following fluence rate increase, and there is a good linear fitting correlation between CL and light dosages.

With the FL spectroscopy, it is shown that FCLA has a FL peak at $515 \mathrm{~nm}$. Figure 6(a) shows the temporal profiles of FL intensity in mouse skin during PDT. On the basis of the

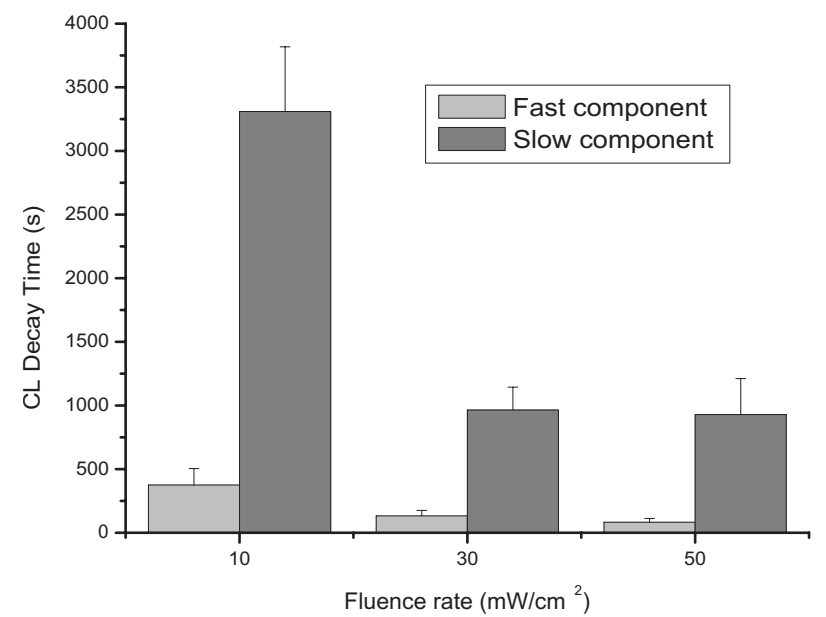

Fig. $3 \mathrm{CL}$ decay time including fast and slow components at fluence rate of 10,30 , and $50 \mathrm{~mW} / \mathrm{cm}^{2}$, respectively (fluence $=30 \mathrm{~J} / \mathrm{cm}^{2}$, $n=7)$. Data are presented as mean \pm SE. 


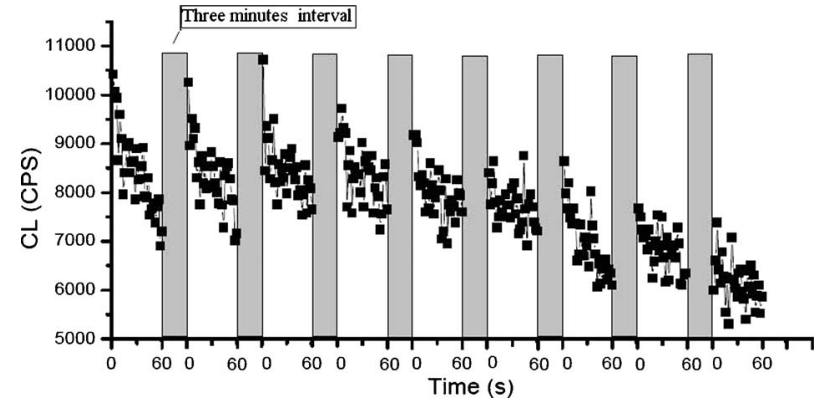

Fig. $4 \mathrm{CL}$ intensity with an interval PDT. Drugs were employed as described in Fig. 2 as and injected into mouse skin. Fluence rate was $50 \mathrm{~mW} / \mathrm{cm}^{2}$. Every irradiation time is $60 \mathrm{~s}$. After a 180-s interval, the next irradiation was carried out. Nine phases of $\mathrm{CL}$ intensity are shown on the chart. Their CL decay time as an exponential decay is $\sim 21 \mathrm{~s}$.

CL correction theory described above, the elimination rate constant $k$ [Eq. (6)] can be obtained with the FL and CL data from the mouse skin [Fig. 6(b)]. The elimination coefficient of a drug is usually a constant in a particular tissue, but the results show that the elimination coefficient calculated based on real-time data did not remain stable and decreased in the later phase during the PDT treatment. This may be partially attributed to vasculature damage that often occurs during a PDT treatment.

FCLA CL is a nonrecyclable process. Our preliminary investigation shows that an oxidized FCLA molecule has a similar spectrum to that of fresh and a higher FL quantum yield. $^{21}$ In our in vivo experiments, we chose to use a FCLA concentration that is adequate to ignore the consumption of the probe, yet not high enough to cause cytotoxicity. ${ }^{22}$

To compensate for the FCLA concentration changes caused by the probe reaction and pharmacokinetics, a correction factor was calculated based on real-time data to quantitatively correct $\mathrm{CL}$ at a given time. In order to accomplish this, the initial concentration of FCLA was first estimated with CL caused by self-oxidation of FCLA of known concentrations in a simulated tissue optical phantom [Fig. 7(a)]. Figure

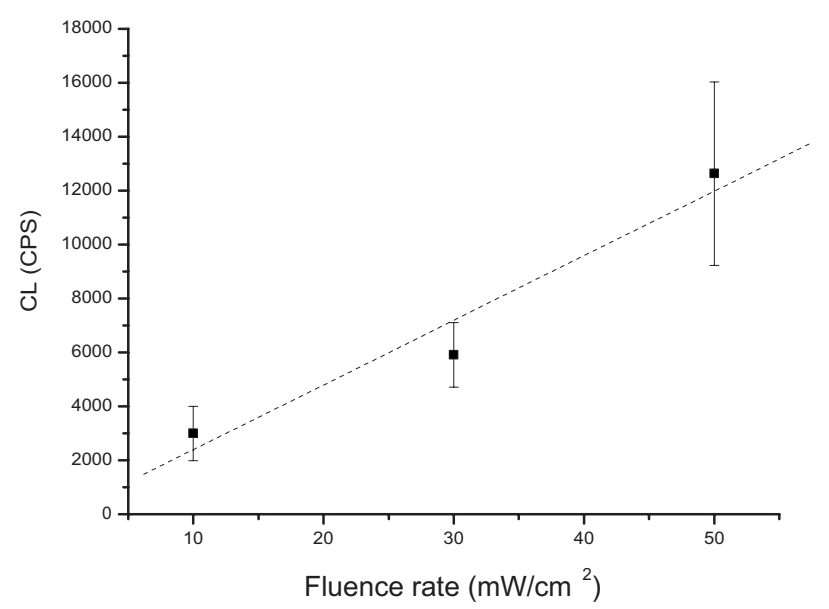

Fig. $5 \mathrm{CL}$ intensity after cessation of PDT irradiation at various fluence rates $\left(10,30,50 \mathrm{~mW} / \mathrm{cm}^{2}\right)$. The data were fitted linear, and the correlation coefficient is 0.98 . Data are present as mean \pm SD.

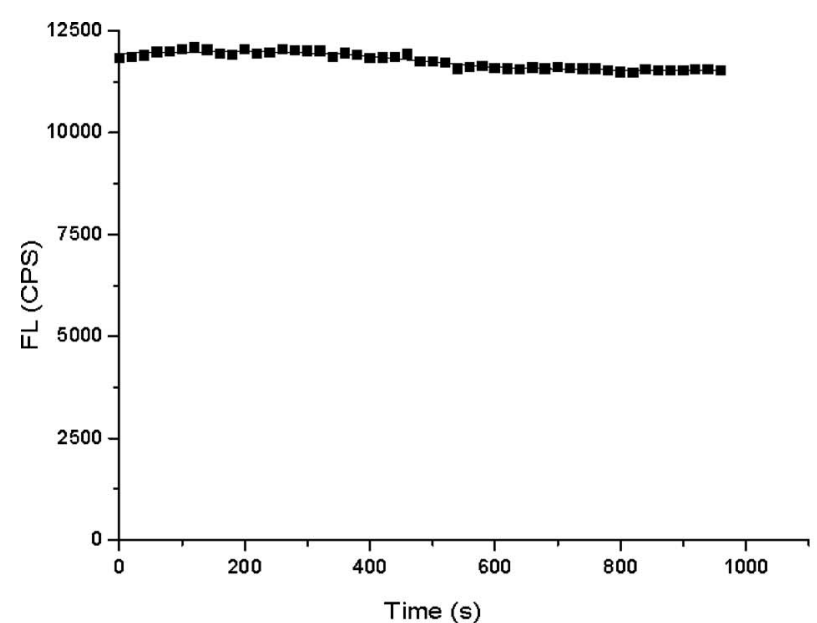

(a)

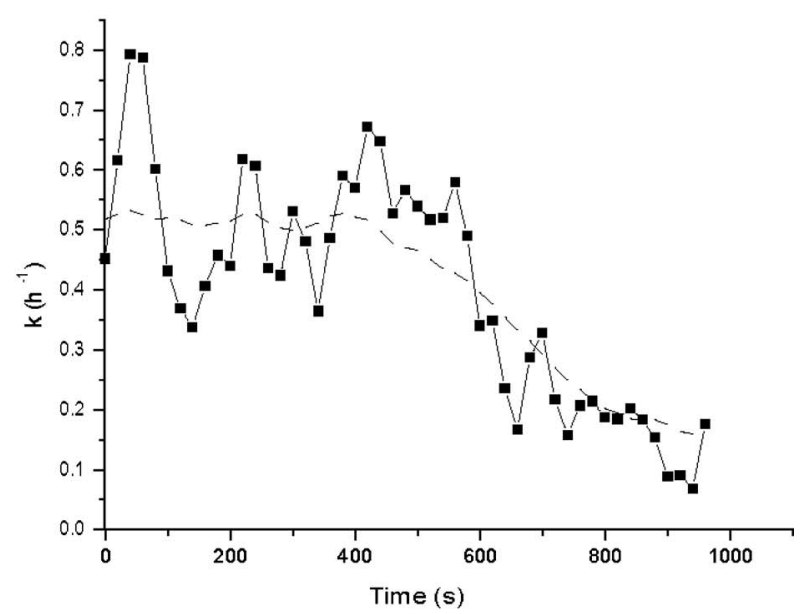

(b)

Fig. 6 (a) FL at $515 \mathrm{~nm}$ of FCLA with a continual photochemical kinetic course. Fluence rate $20 \mathrm{~mW} / \mathrm{cm}^{2}$ for $635-\mathrm{nm}$ laser and $5 \mu \mathrm{W} / \mathrm{cm}^{2}$ for $488-\mathrm{nm}$ laser were used. (b) The first-order elimination coefficient during PDT. The data were calculated with the values of chemiluminescence and FL that were both detected during PDT course [Eq. (6)]. The dashed line is the adjacent averaging line.

7(b) is a representative of CL correction based on an estimated initial FCLA probe concentration and the correction factor $\left[c_{0} / c_{t}\right.$, from Eq. (11), as shown in the inset] based on real-time CL and FL measurement.

\section{Discussion}

FCLA is a highly sensitive chemiluminescence probe for detecting ${ }^{1} \mathrm{O}_{2}$ and $\mathrm{O}_{2^{-}}$. Results from current study have demonstrated the feasibility of in vivo ROS detection with FCLA during PDT. By using a delay CL-detection apparatus, interference from the irradiation light source is effectively eliminated to achieve a higher signal-to-noise ratio for CL acquisition. We have previously reported that cumulative CL acquired during a PDT treatment can be related to the biological outcome of the treatment. ${ }^{19}$ In the current study, the results further show that CL intensity is linearly related to the irradiation light fluence rate (Fig. 5). Given the mechanism of ROS production, this observation strongly suggests that $\mathrm{CL}$ is 


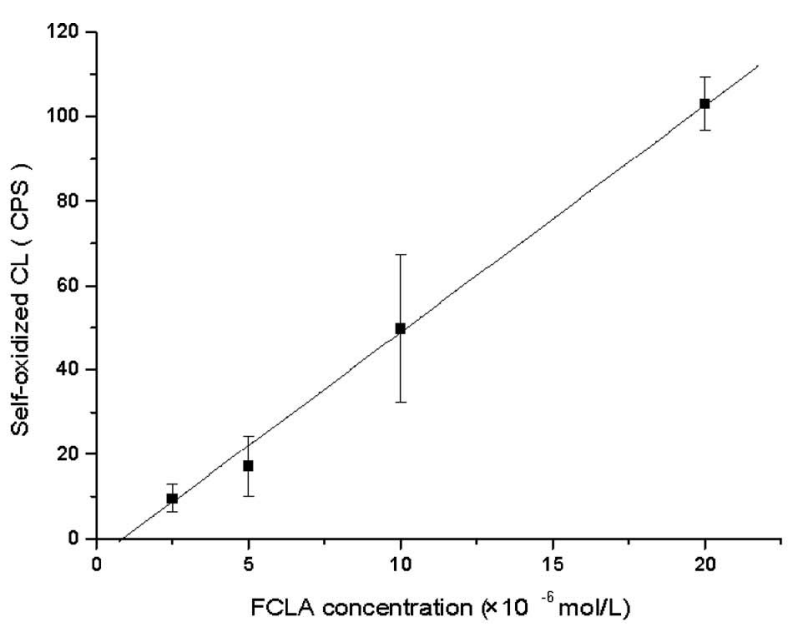

(a)

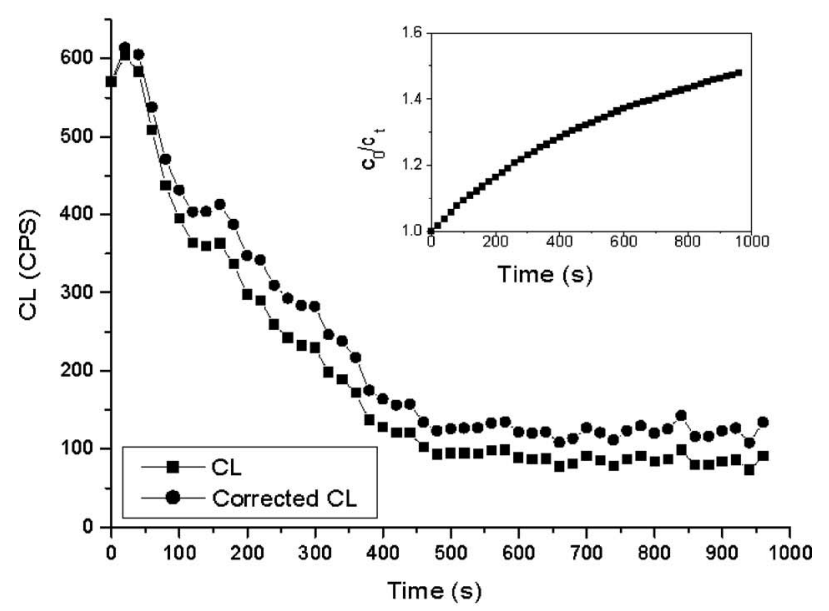

(b)

Fig. 7 (a) The self-oxidized CL of FCLA at various concentrations in the stimulated skin solution $(n=3): C L=5.4 \times[\mathrm{FCLA}], R=0.99$. (b) The $C L$ and the corrected $C L$. The accessorial chart is the dynamic coefficient for $\mathrm{CL}$ correction.

a reasonable marker for monitoring ROS production.

As shown in Fig. 2, CL intensity decreased gradually during PDT. Many factors may contribute to the decrease. Understanding these factors will help us to advance our knowledge and improve ROS detection with CL in PDT. Statistical analysis reveals that the decay time of both fast and slow components of $\mathrm{CL}$ is reasonably consistent, given identical treatment protocols, but varies considerably depending on the irradiation fluence rate. In particular, CL decay time for both fast and slow components decreased rapidly as the irradiation fluence rate increased, but reached a plateau at a higher fluence rate. The rapidly decreasing component of CL is most likely related to the oxygen depletion that occurs during the PDT light-irradiation process. This may include oxygen bleaching as a result of photochemical reactions of both PDT and CL production. ${ }^{23,24}$ Oxygen perfusion from microvasculature is not likely to adequately compensate for this depletion. This may, to a degree, explain the rapid decrease of the fast component of CL decay time. When the irradiation fluence rate is high, the overall oxygen depletion must exceed that which is being supplied. As a result, the CL production rate is governed by the diffusion rate, leading to the observed plateau at the higher irradiation fluence rate. In addition, photosensitizer bleaching during PDT, as reported by various investigators, may also contribute to the changes of CL production. More photosensitizer will be bleached (ROS product reduces) with laser fluence increase. ${ }^{25-28}$ These are all the reasons for a fast component.

By fractionating the irradiation light fluence, tissue reoxygenation was allowed during the intermittent dark period. As shown in Fig. 4, this effectively allows a recovery of the CL production (observed intensity). If oxygen consumption is ignored by only comparing the initial CL, then CL intensity increased linearly with light fluence rates (Fig. 5). These results are all in agreement with the discussion above, that the fast component is attributed to the oxygen depletion from photochemical reactions directly related to the light irradiation. In Fig. 4, even when given a reasonably long dark period for reoxygenation, the CL production rate does not fully return to its previous level but shows a general trend of slowly decreasing. The time constant of this decrease is in general agreement with that of the slow component. It is possible that FCLA probe pharmacokinetics (tissue elimination) played a role in the phenomenon. By alternately monitoring CL and FL during PDT in situ, based on Eq. (11), a correction to the observed CL value was made to compensate for both chemical consumption and tissue elimination of the probe. It was found that these processes had minimal effect on CL production. The results show that the FCLA FL intensity remained stable $1 \mathrm{~h}$ after its initial administration throughout the datacollection period [Fig. 6(a)]. By calculating the FCLA elimination rate, as shown in Fig. 6(b), it is demonstrated that the elimination rate of FCLA from tissue is much slower compared to the observed CL decreasing by either the slow or fast component. This suggests that tissue FCLA concentration remained stable during PDT, and the slow component of CL decreasing is likely due to other factors. A photosensitizer can result in vascular damage during PDT. Although the process is less dramatic compared to PDT treatment that directly targets the vasculature, it has been reported that the vascular damage and subsequent reduction in oxygen supply can occur in PDT as early as $<1 \mathrm{~h}$ after the light irradiation commences. ${ }^{29}$ Of course, the plateau of the slow component when the fluence rate is high might also be the result of vasculature damage.

In conclusion, ROS production is a critical factor in PDT. A ROS-detection system based on the CL probe FCLA was designed and used to monitor ROS in situ during in vivo PDT. By utilizing the characteristic long decaying time of CL and a gated data acquisition scheme, the system achieves a high sensitivity for practical applications. The effect of CL probe concentration on CL during PDT was studied and theoretically modeled by simultaneously monitoring CL and FL of the probe. The results show that, by carefully choosing a probeadministrating protocol, the effect of FCLA pharmacokinetics can be neglected, thus greatly simplifying the procedure for practical applications. It is realized that there is future work necessary to consider photon transmission in biological tissue for a true quantitative measurement of ROS production. The principle and functional apparatus realized in the current work shall provide a solid foundation for in vivo ROS monitoring during PDT. 
Wei et al.: Quantitative measurement of reactive oxygen species in vivo by utilizing...

\section{Acknowledgments}

This research is supported by the National Basic Research Program of China (Grant No. 2010CB732602), the Program for Changjiang Scholars and Innovative Research Team in University (Grant No. IRT0829), and the National Natural Science Foundation of China (Grant No. 30870676 and No. 30870658).

\section{References}

1. W. M. Sharman, C. M. Allen, and J. E. Vanlier, "Photodynamic therapeutics: basic principles and clinical application," Drug Discovery Today 4, 507-517 (1999).

2. N. L. Oleinick, R. L. Morris, and I. Belichenko, "The role of apoptosis in response to photodynamic therapy: what, where, why, and how," Photochem. Photobiol. Sci. 1, 1-21 (2002).

3. H. Kostron, A. Obwegeser, and R. Jakober, "Photodynamic therapy in neurosurgery: a review," J. Photochem. Photobiol., B 36, 157-168 (1996).

4. S. B. Brown, E. A. Brown, and I. Walker, "The present and future role of photodynamic therapy in cancer treatment," Lancet. Oncol. 5 , 497-508 (2004).

5. J. Yamamoto, S. J. Yamamoto, T. Hirano, S. Y. Li, M. Koide, E. Kohno, M. Okada, C. Inenaga, T. Tokuyama, N. Yokota, S. Terakawa, and H. Namba, "Monitoring of singlet oxygen is useful for predicting the photodynamic effects in the treatment for experimental glioma," Clin. Cancer Res. 12(23), 7132-7137( 2006).

6. M. T. Jarvi, M. J. Niedre, M. S. Patterson, and B. C. Wilson, "Singlet oxygen luminescence dosimetry (SOLD) for photodynamic therapy: current status, challenges and future prospects," Photochem. Photobiol. 82, 1198-1210 (2006).

7. C. Dodeigne, L. Thunus, and R. Lejeune, "Chemiluminescence as a diagnostic tool. a review," Talanta 51, 415-439 (2000).

8. F. Wu, Y. Huang, and C. Huang, "Chemiluminescence biosensor system for lactic acid using natural animal tissue as recognition element," Biosens. Bioelectron. 21(3), 518-522 (2005).

9. M. M. Pelletier, P. Kleinbongard, L. Ringwood, R. Hito, C. J. Hunter, A. N. Schechter, M. T. Gladwin, T. Mark, and A. Dejam, "The measurement of blood and plasma nitrite by chemiluminescence: pitfalls and solutions," Free Radic Biol. Med. 41(4), 541-548 (2006).

10. X. H. Li, G. X. Zhang, H. M. Ma, D. Q. Zhang, J. Li, and D. B. Zhu, "4,5-Dimethylthio-4 $\phi$-[2-(9-anthryloxy)ethylthio]tetrathiafulvalene, a highly selective and sensitive chemiluminescence probe for singlet oxygen," J. Am. Chem. Soc. 126, 11543-11548 (2004).

11. M. Mitani, Y. Yokoyama, S. Ichikawa, H. Sawada, T. Matsumoto, K. Fujimori, and M. Kosugi "Determination of horseradish peroxidase concentration using the chemiluminescence of Cypridina luciferin analogue," J. Biolumin. Chemilumin. 9, 355-361 (1994).

12. J. Wang and D. Xing, "Detection of vitamin C-induced singlet oxygen formation in oxidized LDL using MCLA as a chemiluminescence probe," Acta Biochim Biophys Sin. 34, 11-15 (2002).

13. S. Sun, X. H. Li, G. X. Zhang, H. M. Ma, D. Q. Zhang, and Z. J. Bao, "Determination of H2O2-dependent generation of singlet oxygen from human saliva with a novel chemiluminescence probe," Biochim. Biophys. Acta 1760(3), 440-444 (2006).
14. Y. H. He, D. Xing, S. C. Tan, Y. H. Tang, and K. Ueda, "In vivo sonoluminescence imaging with the assistance of FCLA," Phys. Med. Biol. 47, 1535-1541 (2002).

15. Y. F. Qin, D. Xing, S. M. Luo, J. Zhou, X. Y. Zhong, and Q. Chen, "Feasibility of using fluoresceinyl cypridina luciferin analog in a novel chemiluminescence method for real-time photodynamic therapy dosimetry," Photochem. Photobiol. 81(6), 1534-1538 (2005).

16. W. Xu, Y. C. Wei, D. Xing, and Q. Chen, "A novel chemiluminescence technique for quantitative measurement of low concentration human serum albumin," Anal. Sci. 24, 115-119 (Jan 2008).

17. Y. C. Wei, J. Zhou, D. Xing, and Q. Chen, "In vivo monitoring of singlet oxygen using delayed chemiluminescence during photodynamic therapy," J. Biomed. Opt. 12(1), 014002 (2007).

18. I. Bronshtein, S. Aulova, and A. Juzeniene, "In vitro and in vivo photosensitization by protoporphyrins prossessing different lipophilicities and vertical lacalizaotion in the membrane," Photochem. Photobiol. 82, 1319-1325 (2006).

19. Y. C. Wei, D. Xing, S. M. Luo, W. Xu, and Q. Chen, "Monitoring singlet oxygen in situ with delayed chemiluminescence to deduce the effect of photodynamic therapy," J. Biomed. Opt. 13(2), 024023 (2008).

20. S. Müller, H. Walt, D. Dobler-Girdziunaite, D. Fiedler, and U. Haller, "Enhanced photodynamic effects using fractionated laser light," $J$. Photochem. Photobiol., B 42(1), 67-70 (1998).

21. Y. C. Wei, S. M. Luo, and W. Xu, "Detection of singlet oxygen and superoxide anion with the fluorescence of FCLA," Spectrosc. Spectral Anal. 28(11), 2633-2635 (2008)

22. Y. X. Wu and D. Xing, "Permeating efficiency and localization of FCLA and HpD through membrane of lung cancer cell," Acta Laser Bio. Sin. 14(4), 287-292 (2005).

23. J. H. Woodhams, L. Kunz, S. G. Bown, and A. J. MacRobert, "Correlation of real-time haemoglobin oxygen saturation monitoring during photodynamic therapy with microvascular effects and tissue necrosis in normal rat liver," Br. J. Cancer 91(4), 788-794 (2004).

24. M. Seshadri, D. A. Bellnier, L. A. Vaughan, J. A. Spernyak, R. Mazurchuk, T. H. Foster, and B. W. Henderson, "Light delivery over extended time periods enhances the effectiveness of photodynamic therapy," Clin. Cancer Res. 14(1), 2796-2805 (2008).

25. R. Bonnett and G. Martinez "Photobleaching of sensitisers used in photodynamic therapy," J. Tetrahedron 57, 9513-9547 (2001).

26. I. A. Boere, D. J. Robinson, H. S. De Bruijn, J. Van Den Boogert, H. W. Tilanus, H. J. C. M. Sterenborg, and W. F. De Bruin, "Monitoring in situ dosimetry and protoporphyrin IX fluorescence photobleaching in the normal rat esophagus during 5-aminolevulinic acid photodynamic therapy," Photochem. Photobiol. 78(3), 271-277 (2003).

27. B. W. McIlroy, T. S. Mann, J. S. Dysart, and B. C. Wilson, "The effects of oxygenation and photosensitizer substrate binding on the use of fluorescence photobleaching as a dose metric for photodynamic therapy," Vib. Spectrosc. 28, 25-35 (2002).

28. J. C. Finlay, S. Mitra, M. S. Patterson, and T. H. Foster, "Photobleaching kinetics of photofrin in vivo and in multicell tumour spheroids indicate two simultaneous bleaching mechanisms," Phys. Med. Biol. 49, 4837-4860 (2004).

29. G. Yu, T. Durduran, C. Zhou, H. Wang, M. E. Putt, H. M. Saunders, C. M. Sehgal, E. Glatstein, A. G. Yodh, and T. M. Busch, "Noninvasive monitoring of murine tumor blood flow during and after photodynamic therapy provides early assessment of therapeutic efficacy," Clin. Cancer Res. 11(9), 3543-3552 (2005). 